\title{
2219. Free vibration analysis of tapered columns under self-weight using pseudospectral method
}

\author{
Gopinathan Sudheer ${ }^{1}$, Pillutla Sri Harikrishna ${ }^{2}$, Yerikalapudy Vasudeva Rao $^{3}$ \\ ${ }^{1}$ GVP College of Engineering for Women, Visakhapatnam, India \\ ${ }^{2}$ GITAM University, Visakhapatnam, India \\ ${ }^{3}$ IIT Bhubaneswar, Bhubaneswar, India \\ ${ }^{1}$ Corresponding author \\ E-mail: ${ }^{1} g$ _sudheer@hotmail.com, ${ }^{2}$ harikrishna.ps@gmail.com, ${ }^{3}$ ryvasudeva@iitbbs.ac.in \\ Received 18 April 2016; received in revised form 10 September 2016; accepted 27 October 2016 \\ DOI https://doi.org/10.21595/jve.2016.17089
}

\begin{abstract}
This paper deals with the vibration of tapered column which is affected by gravity using a pseudospectral formulation. The formulation is simple and easy-to-implement and is capable of dealing with different end conditions. Numerical examples of the effects of taper, cross section shapes and gravity on the vibration of columns are illustrated. The effectiveness of the pseudospectral method for vibration analysis of tapered heavy columns is validated by comparing the results with numerical techniques such as the numerical initial value method and differential quadrature method.
\end{abstract}

Keywords: pseudospectral, non-uniform, columns, taper, gravity.

\section{Introduction}

Elastic columns are a class of important structural components that find wide applications in civil, mechanical and aerospace engineering fields [1]. The strength of an elastic column basically depends on its material and geometrical properties. The material selection and Young's modulus determine whether a column has material non-linearity while the geometric non-linearity arises from non-uniform cross-sectional areas [2]. In conventional column vibration and buckling problems, the self-weight is often neglected and when taken into consideration, the column is referred to as a heavy column [3]. The standing heavy column is fundamental in mechanics and models tall structures and free-standing antennas [4].

Greenhill [5], using Bessel's functions, first investigated the stability of a uniform column due to its own weight. Schafer [6] studied the effect of self-weight on the natural frequencies of a hanging cantilever beam using the Rayleigh-Ritz method. The finite element method was used by Yokoyama [7] to investigate the vibration characteristics of uniform hanging beams under gravity. Virgin et al. [8] performed analytical and experimental studies on the effect of gravity on the vibration of vertical cantilevers. Duan and Wang [3] presented analytical solutions for the buckling of columns including self-weight. Okay et al. [9] applied the variational iteration method to determine the buckling loads and mode shapes of heavy columns under its own weight. An analytic method involving the Fredholm integration method was used by Huan and $\mathrm{Li}$ [10] to analyze the buckling behavior of axially nonuniform graded columns. The differential quadrature (DQ) method was used by Mahmoud et al [11] to investigate the effect of column geometry on the natural frequencies and mode shapes. Taha and Essam [12] used the DQ method to study the stability behavior and free vibration of axially loaded tapered columns with elastic end restraints. Recently, Wang [13] used a numerical initial value method to study the influence of gravity as well as taper on the vibration of a standing column.

Although many methods have been presented to analyze problems concerned with taper and self-weight, most of them apply to specific cases determined by the form of the equations. This study presents a simple numerical technique that is capable of handling different cases using the pseudospectral formulation. In terms of effective mathematical techniques, Pesudospectral (PS) methods have been used in recent years for structural engineering analysis. Lee and Schultz [14] applied the Chebyshev PS method to solve the vibration of Timoshenko beams and Mindlin plates. 
Yagci et al. [15] used a spectral Chebyshev technique for solving linear and nonlinear beam equations. Sari and Butcher [16] used the PS method for the free vibration analyses of non-rotating and rotating Timoshenko beams with damaged boundaries. In [17], the PS method is used to investigate the dynamic response of Timoshenko beams made of functionally graded materials. A Chebyshev PS method is presented in [18] for the static analysis of the geometrically exact beams undergoing large deflections. However, to the author's knowledge no analytical solution exists for the important problem of the vibration of a standing heavy tapered column. A numerical initial value method proposed in [13] that combines the initial value method [19] with Runge-Kutta method and bisection method is the only available technique present in literature. The important problem demands techniques that are easy to implement and computationally inexpensive.

The intention of this work is to explore the application of a novel formulation of the Chebyshev Pseudospectral method to the vibration analysis of heavy and tapered columns. The method is first validated by computing the frequencies of vibration of non-uniform beams where their density and the flexural rigidity vary along the longitudinal axis. The influence of gravity, taper and gravity and taper on the vibrations of columns is then analyzed and the results are compared with those obtained using exact solutions [20], a Differential Quadrature Method [11] and a numerical initial value method [13].

\section{Equations}

The vibration of long and slender beams/columns is an important problem in applied mechanics and are generally modeled by the Euler-Bernoulli beam theory [21]. Assuming the effects of rotational inertia and transverse shear deformation to be negligible, the equation for small vibrations of a non-uniform Euler Bernoulli column subjected to an axial force $F$ is given by [13]:

$$
\frac{\partial^{2}}{\partial x^{2}}\left(E I(x) \frac{\partial^{2} y}{\partial x^{2}}\right)+\frac{\partial}{\partial x}\left(F(x) \frac{\partial y}{\partial x}\right)+\rho(x) \frac{\partial^{2} y}{\partial t^{2}}=0,
$$

where $(x, y)$ are the longitudinal and transverse coordinates of the column with the origin at the base, $E I$ is the flexural rigidity, $\rho$ is the mass per length and $t$ is the time. For a standing column of height $L$, axial force is given by:

$F=g \int_{x}^{L} \rho(x) d x$

where $g$ is the acceleration due to gravity. Introducing $y(x, t)=w(x) e^{i k t}, E I(x)=E I_{o} l(x)$, $\rho(x)=\rho_{o} r(x)$, where $E I_{o}$ and $\rho_{o}$ are the maximum values of flexural rigidity and mass per length occurring at the base $x=0$ and $k$ is the frequency of vibration. Normalizing all length by $L$ Eq. (1), becomes:

$\frac{d^{2}}{d x^{2}}\left[l(x) \frac{d^{2} w}{d x^{2}}\right]+\beta \frac{d}{d x}\left[\int_{x}^{1} r(x) \frac{d w}{d x}\right]=\omega^{2} r(x) w$,

where $\omega=k L^{2} \sqrt{\rho_{o} / E I_{o}}$ and $\beta=g \rho_{o} L^{3} / E I_{o}$.

Eq. (3) does not have a closed form solution even for a uniform beam/column [21]. Assuming that the column has linear taper with the rigidity and density varying as $l(x)=(1-c x)^{m}$, $r(x)=(1-c x)^{n}$ where $m, n$ are positive constants and $0 \leq c \leq 1$ representing the degree of taper, Eq. (3) becomes: 


$$
\frac{d^{4} w}{d x^{4}}+b_{1}(x) \frac{d^{3} w}{d x^{3}}+b_{2}(x) \frac{d^{2} w}{d x^{2}}+b_{3}(x) \frac{d w}{d x}+b_{4}(x) w=0,
$$

where:

$b_{1}(x)=-\frac{2 m c}{1-c x}, \quad(c \neq 0)$,

$b_{2}(x)=\frac{m(m-1) c^{2}}{(1-c x)^{2}}+\frac{\beta\left\{(1-c x)^{n+1}-(1-c)^{n+1}\right\}}{c(n+1)(1-c x)^{m}}=\beta(1-x), \quad(c=0)$,

$b_{3}(x)=-\beta(1-c x)^{n-m}$,

$b_{4}(x)=-\omega^{2}(1-c x)^{n-m}$.

For clamped-free (C-F) columns with clamped end $x=0$ and free end $x=1$, the boundary conditions can be written as:

$w=\frac{d w}{d x}=0, \quad x=0$,

$\frac{d^{2} w}{d x^{2}}=0, \quad \frac{d}{d x}\left[l(x) \frac{d^{2} w}{d x^{2}}\right]=0, \quad x=1$.

If $c=0$, the column is uniform and for $c=1$, the column has a pointy tip.

For $c \neq 1$, Eq. (6) reduces to:

$\frac{d^{2} w}{d x^{2}}=0, \quad \frac{d^{3} w}{d x^{3}}=0, \quad x=1$.

Eq. (4) is solved subject to the boundary conditions given by Eq. (5) and Eq. (6) for different values of $m, n$. Though the equations can be solved for general values of $m, n$, we consider some special cases which correspond to those shown in Fig. 1. In the case of a solid tapered column of circular cross section, $m=4, n=2$, while for a solid column of constant thickness and tapered sides $m=1, n=1$ or $m=3, n=1$. If the column vibrates about the axis A-A which is perpendicular to the thickness direction $m=1, n=1$ and if the column vibrates about the axis B-B which is parallel to the thickness direction $m=3, n=1$ [13].

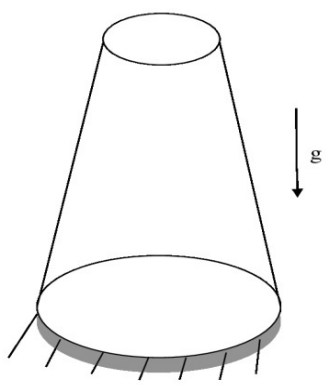

a)

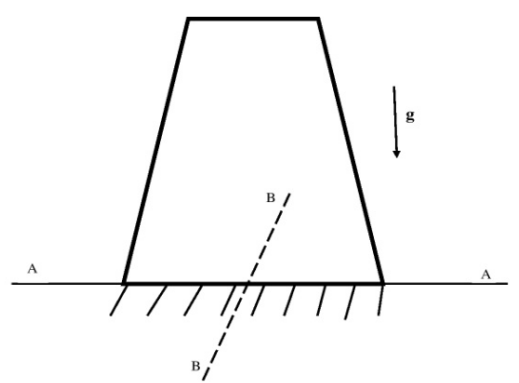

b)

Fig. 1. a) Tapered column of circular cross section, b) solid column of constant thickness and tapered sides

\subsection{Solution procedure}

In Chebyshev PS method, the Chebyshev polynomials are employed as the trial functions for the discretization of the unknown function namely $w$ and the Gauss-Chebyshev-Lobatto points are employed as the collocation points at which the residuals are minimized. The physical domain 
$0 \leq x \leq 1$ is transformed into $-1 \leq X \leq 1$ by the transformation $X=2 x-1$. With this transformation Eq. (4) reduces to:

$\frac{d^{4} w}{d X^{4}}+B_{1}(X) \frac{d^{3} w}{d X^{3}}+B_{2}(X) \frac{d^{2} w}{d X^{2}}+B_{3}(X) \frac{d w}{d X}+B_{4}(X) w=0$,

where $B_{i}(X)=b_{i}(x) / 2^{i}, i=1,2,3,4$.

We assume:

$w=\sum_{k=0}^{N} a_{k} T_{k}(X)$,

where $a_{k},(k=0, \ldots, N)$ are unknown constants and $T_{k}(X)(k=0,1, \ldots, N)$ are Chebyshev polynomials defined by [22] $T_{k}(X)=\cos \left(k \cos ^{-1}(X)\right)=\cos (k \theta)$ for $k=0,1,2, \ldots$, where $\theta=\cos ^{-1}(X)$.

The transformation $X=\cos \theta$ converts the Chebyshev series into a Fourier cosine series. In the proposed methodology, we compute the basis functions and their derivatives using trigonometric functions:

i.e. $X=\cos \theta \leftrightarrow \theta=\cos ^{-1}(X), \quad X \in\left[\begin{array}{ll}-1 & 1\end{array}\right], \quad t \in\left[\begin{array}{ll}0 & \pi\end{array}\right]$.

The elementary identity:

$\frac{d}{d X} \leftrightarrow\left(-\frac{1}{\sin \theta}\right) \frac{d}{d \theta}$

is repeatedly applied to convert Eq. (8) into an equivalent differential equation on $\theta \in[0 \pi]$. As $\theta \rightarrow 0, \pi$ the derivatives are evaluated using the Taylor expansions of both numerator and denominator about their common zero. Substituting the value of $w$ given by Eq. (9) into Eq. (8) and using Eq. (10), Eq. (11) the equivalent differential equation is collocated at:

$\theta_{i}=\frac{(N-i) \pi}{N}, \quad i=1, \ldots, N-3, \quad(0, \pi)$,

yielding a system of $(N-3)$ equations in $N+1$ unknowns $a_{k}$. Imposing the boundary conditions given by Eq. (5), Eq. (6) we get a system of 4 equations in $(N+1)$ unknowns. The resulting $(N+1)$ by $(N+1)$ system of equations is expressed as a matrix eigenvalue problem and solved using a standard eigensolver.

\section{Numerical results and discussion}

In this section, we study the convergence behavior of the PS method first and then consider some numerical examples to validate the efficiency of the Pseudospectral method. The first numerical example is devoted to a beam with constant thickness and linearly tapered width with clamped base and no tip mass. The second is concerned with the vibration of a hanging uniform column under self-weight. The third example considers the free vibration of non-uniform column with no self-weight. The last example concerns the influence of gravity and taper on the vibration of a standing column.

\subsection{Convergence behavior of PS method}

As a case study, the convergence behavior of the non-dimensional frequency parameter $(\omega)$ 
for the first five modes of a non-uniform beam whose cross section is an open web or tower type [20] is considered. To highlight this, we consider the clamped-free boundary condition with a taper ratio of $c=0.1$ in the absence of gravity. Taking the exact values of $(\omega)$ given in [20] as base values, we compute the values of $(\omega)$ for $N$ varying from 20 to 28 . The results obtained to six-digit precision are presented in Table 1. We observe from the table that the converged results have good accuracy in comparison with those of [20].

Table 1. Non-dimensional vibration frequencies for $m=4, n=0$

\begin{tabular}{|c|c|c|c|c|c|c|}
\hline \multirow{2}{*}{$(\omega)$} & \multicolumn{6}{|c|}{$N$} \\
\cline { 2 - 7 } & 20 & 22 & 24 & 26 & 27 & 28 \\
\hline$\omega_{1}$ & 3.376722 & 3.376722 & 3.376722 & 3.376722 & 3.376722 & 3.376722 \\
\hline$\omega_{2}$ & 20.248149 & 20.248149 & 20.248149 & 20.248149 & 20.248149 & 20.248149 \\
\hline$\omega_{3}$ & 55.966597 & 55.966595 & 55.966595 & 55.966595 & 55.966595 & 55.966595 \\
\hline$\omega_{4}$ & 109.302542 & 109.301970 & 109.301978 & 109.301978 & 109.301978 & 109.301978 \\
\hline$\omega_{5}$ & 180.403245 & 180.432576 & 180.430075 & 180.430210 & 180.430205 & 180.430205 \\
\hline
\end{tabular}

\subsection{Vibration of a class of Non-uniform beams in the absence of gravity}

In [20], new exact solutions were presented for a class of non-uniform beams whose density and flexural rigidity vary along the longitudinal axis. To assess the numerical accuracy of the proposed PS method, we obtain the numerical solutions for a class of non-uniform beams presented in [20]. Ignoring rotational inertia and shear deformation, the Euler-Bernoulli small deflection beam equation is obtained by taking $F \equiv 0$ in Eq. (1). To test the validity of the method for different end conditions we consider Clamped-Free (C-F), Pinned-Pinned (P-P) and Free-Free (F-F) end conditions. At clamped and free ends the boundary conditions can be used from Eqs. (5-6). For a pinned end the conditions are $w=0, d^{2} w / d x^{2}=0$.

The computations in the PS method (PSM) are carried out using $N=30$ and the results obtained are presented in Table 2 along with the exact values [20]. The exact values given in [20] are obtained through power function solutions. The results are presented in the form of tables to highlight the numerical accuracy and for easy comparison.

Table 2. Non-dimensional vibration frequencies for $m=4, n=0$

\begin{tabular}{|c|c|c|c|c|c|c|}
\hline Taper ratio $c$ & \multicolumn{2}{|c|}{0.1} & \multicolumn{2}{c|}{0.3} & \multicolumn{2}{c|}{0.7} \\
\hline Boundary conditions & Wang [20] & PSM & Wang [20] & PSM & Wang [20] & PSM \\
\hline \multirow{5}{*}{ C-F } & 3.3767 & 3.376722 & 3.0751 & 3.075097 & 2.3151 & 2.315075 \\
\cline { 2 - 7 } & 20.248 & 20.248149 & 16.680 & 16.680409 & 9.3906 & 9.390641 \\
\cline { 2 - 7 } & 55.367 & 55.966595 & 44.733 & 44.733218 & 22.898 & 22.897800 \\
\cline { 2 - 7 } & 109.30 & 109.301978 & 86.631 & 86.630457 & 42.975 & 42.974975 \\
\cline { 2 - 7 } & 180.43 & 180.430205 & 142.50 & 142.494970 & 69.698 & 69.698967 \\
\hline \multirow{5}{*}{ F-F } & 20.162 & 20.161464 & 15.890 & 15.889909 & 7.9004 & 7.900440 \\
\cline { 2 - 7 } & 55.566 & 55.566239 & 43.715 & 43.714562 & 21.303 & 21.302894 \\
\cline { 2 - 7 } & 108.92 & 108.923974 & 86.625 & 85.625417 & 41.369 & 41.369409 \\
\cline { 2 - 7 } & 180.05 & 180.050691 & 141.49 & 141.487640 & 68.084 & 68.085117 \\
\cline { 2 - 7 } & 268.96 & 268.959692 & 211.31 & 211.313335 & 101.46 & 101.465340 \\
\hline \multirow{5}{*}{ P-P } & 8.8895 & 8.889481 & 6.9698 & 6.969833 & 3.2686 & 3.268554 \\
\cline { 2 - 7 } & 35.570 & 35.569613 & 27.985 & 27.984517 & 13.647 & 13.646465 \\
\cline { 2 - 7 } & 80.026 & 80.026031 & 62.915 & 62.914773 & 30.430 & 30.430199 \\
\cline { 2 - 7 } & 142.26 & 142.263141 & 111.80 & 111.800265 & 53.836 & 53.836215 \\
\cline { 2 - 7 } & 222.28 & 222.281555 & 174.65 & 174.646486 & 83.894 & 83.894116 \\
\hline
\end{tabular}

\subsection{Vibration of a uniform column under self-weight}

The equation for the vibration of a uniform column under self-weight is obtained by taking $c=0$ in Eq. (4). The present scheme is applied to solve the resulting equation using $N=25$. The 
results obtained are compared with the values given in [4]. It is to be noted that negative values of $\beta$ denotes a hanging column. The first two square of non-dimensional frequency values $\left(\omega^{2}\right)$ obtained using the present method with the base fixed and the top experiencing zero moment and shear is given in Table 3. The closeness of the results obtained using PSM with that of the results obtained using the method of [19] is seen in the table.

Table 3. Square of Non-dimensional frequency values of a column under self-weight

\begin{tabular}{|c|c|c|c|c|c|c|c|c|}
\hline \multirow{3}{*}{$\omega^{2}$} & \multicolumn{9}{|c|}{$\beta$} \\
\cline { 2 - 9 } & \multicolumn{2}{|c|}{0} & \multicolumn{2}{|c|}{-20} & \multicolumn{2}{|c|}{-50} & \multicolumn{2}{c|}{-100} \\
\cline { 2 - 9 } & Wang [4] & PSM & Wang [4] & PSM & Wang [4] & PSM & Wang [4] & PSM \\
\hline$\omega_{1}^{2}$ & 12.362 & 12.362363 & 43.53 & 43.530595 & 89.65 & 89.653010 & 165.6 & 165.604518 \\
\hline$\omega_{2}^{2}$ & 485.50 & 485.518818 & 657.7 & 657.648489 & 913.1 & 913.0784445 & 1333 & 1332.919282 \\
\hline
\end{tabular}

\subsection{Free Vibration of non-uniform column}

The governing equation of a non-uniform column varying with bending stiffness given by $1+\alpha x$ as in [11] under no self-weight is obtained by taking $n=0, m=1, \beta=0$ and $c=-\alpha$ in Eqn. (4). In [11], the free vibration of non-uniform column was considered efficiently using the Differential Quadrature Method(DQM). Two variants of DQM namely the modifying weighting coefficient matrices (MWCM) method and substituting boundary conditions into governing equations (SBCGE) techniques were used to treat different types of boundary conditions in [11]. The simple-simple (S-S) supports/pinned-pinned (P-P) supports and clamped-simple (C-S) supports at bottom and top were treated using MWCM technique while the clamped-clamped (C-C) supports and clamped-free (C-F) supports were treated using SBCGE technique. However, the present Pseudospectral formulation is capable of treating the different boundary conditions with ease to compute the non-dimensional frequencies $(\omega)$ of non-uniform columns. The computed first three frequency values for $\alpha=-0.5,0.5$ in the case of S-S and C-C supports are presented in Table 4 along with the corresponding values of [11]. It is to be noted that the computations were carried out using $N=15$ as in [11] for a fair comparison. The results show that the present formulation of PS method is an efficient method in solving the free vibration of non-uniform columns with good accuracy.

Table 4. Non-dimensional frequency values of non-uniform column

\begin{tabular}{|c|c|c|c|c|c|c|c|c|}
\hline \multirow{2}{*}{$\begin{array}{c}\text { Non- } \\
\text { dimensional } \\
\text { frequency } \\
\text { values }\end{array}$} & \multicolumn{2}{|c|}{$\alpha=-0.5$} & \multicolumn{2}{c|}{$\alpha=0.5$} & \multicolumn{2}{c|}{$\alpha=-0.5$} & \multicolumn{2}{c|}{$\alpha=0.5$} \\
\cline { 2 - 9 } & $\begin{array}{c}\text { MWCM } \\
{[11]}\end{array}$ & PSM & $\begin{array}{c}\text { MWCM } \\
{[11]}\end{array}$ & PSM & $\begin{array}{c}\text { SBCGE } \\
{[11]}\end{array}$ & PSM & $\begin{array}{c}\text { SBCGE } \\
{[11]}\end{array}$ & PSM \\
\hline$\omega_{1}$ & 8.479 & 8.479450 & 11.003 & 11.003523 & 19.098 & 19.098602 & 24.888 & 24.888283 \\
\hline$\omega_{2}$ & 33.834 & 33.834311 & 43.976 & 43.976308 & 52.709 & 52.709121 & 68.633 & 68.633917 \\
\hline$\omega_{3}$ & 76.065 & 76.065006 & 98.919 & 98.919180 & 103.38 & 103.383220 & 134.57 & 134.575354 \\
\hline
\end{tabular}

\subsection{Vibration of a standing tapered heavy column}

In [13], the stability and natural vibration of a standing tapered vertical column under its own weight is studied. The method consists in using a simple initial value method combined with interpolation using the Runge-Kutta method to obtain the frequencies of vibration. The present Pseudospectral method is much simpler to implement for computer usage. Though the method is suitable for general values of cross-section shape parameters $(m, n)$, we consider the values $(3$, $1),(1,1)$ and $(4,2)$ for a fair comparison. The non-dimensional vibration frequencies are computed for the taper values of $c=0.1,0.3$ and 0.7 with the gravity parameter $(\beta)$ taking the values $0,2.5$ and 7.5. The computations in the PSM are carried out using $N=25$. The results obtained are presented in Tables 5-7 and are compared with the corresponding values given in [13]. In [13], there is a misprint in the 2nd frequency values corresponding to $c=0.7, \beta=2.5$ and $c=0.3$, 
$\beta=7.5$ for $m=3, n=1$ and the correct values obtained using PSM are given in Table 5. It is observed that as the gravity effect $(\beta)$ increases, the frequencies decrease until the fundamental frequency is almost zero, at which stage the column buckles. In addition, the closeness of the values with those of [13] brings out the simplicity and accuracy of the present method.

Table 5. Non-dimensional frequencies for $m=3, n=1$

\begin{tabular}{|c|c|c|c|c|c|c|}
\hline \multirow{3}{*}{$\beta$} & \multicolumn{7}{|c|}{$c$} \\
\cline { 2 - 7 } & \multicolumn{2}{|c|}{0} & \multicolumn{2}{c|}{0.3} & \multicolumn{2}{c|}{0.7} \\
\cline { 2 - 7 } & Wang [13] & PSM & Wang [13] & PSM & Wang [13] & PSM \\
\hline \multirow{4}{*}{0} & 3.5587 & 3.558702 & 3.667 & 3.666749 & 4.0817 & 4.081714 \\
\cline { 2 - 7 } & 21.338 & 21.338102 & 19.889 & 19.880606 & 16.625 & 16.625269 \\
\cline { 2 - 7 } & 58.980 & 58.979904 & 53.322 & 53.322198 & 40.588 & 40.587991 \\
\hline \multirow{3}{*}{2.5} & 2.9485 & 2.948465 & 3.0621 & 3.062087 & 3.4971 & 3.497114 \\
\cline { 2 - 7 } & 20.837 & 20.836806 & 19.369 & 19.368556 & 14.085 & 16.085256 \\
\cline { 2 - 7 } & 58.470 & 58.469500 & 52.806 & 52.805953 & 40.051 & 40.051452 \\
\hline \multirow{3}{*}{7.5} & 0.8466 & 0.846644 & 1.0938 & 1.093815 & 1.8155 & 1.815472 \\
\cline { 2 - 7 } & 19.794 & 19.794401 & 19.300 & 18.300150 & 14.947 & 14.946583 \\
\cline { 2 - 7 } & 57.433 & 57.432830 & 51.756 & 51.755963 & 38.955 & 38.954952 \\
\hline
\end{tabular}

Table 6. Non-dimensional frequencies for $m=1, n=1$

\begin{tabular}{|c|c|c|c|c|c|c|}
\hline \multirow{4}{*}{$\beta$} & \multicolumn{9}{|c|}{$c$} & \multicolumn{2}{c|}{0.7} \\
\cline { 2 - 7 } & \multicolumn{2}{|c|}{0.1} & \multicolumn{2}{c|}{0.3} & \multicolumn{2}{|c|}{} \\
\cline { 2 - 7 } & Wang [13] & PSM & Wang [13] & PSM & Wang [13] & PSM \\
\hline \multirow{4}{*}{0} & 3.6310 & 3.631027 & 3.9160 & 3.916033 & 4.9317 & 4.931642 \\
\cline { 2 - 7 } & 22.254 & 22.254029 & 22.786 & 22.785958 & 24.687 & 24.687279 \\
\cline { 2 - 7 } & 61.910 & 61.909628 & 62.463 & 62.436120 & 64.527 & 64.526628 \\
\hline \multirow{3}{*}{2.5} & 3.0376 & 3.037632 & 3.3638 & 3.363823 & 4.4793 & 4.479281 \\
\cline { 2 - 7 } & 21.771 & 21.770841 & 22.333 & 22.332727 & 24.315 & 24.315225 \\
\cline { 2 - 7 } & 61.416 & 61.416302 & 61.976 & 61.975560 & 64.153 & 64.152600 \\
\hline \multirow{3}{*}{7.5} & 1.1326 & 1.132558 & 1.8024 & 1.802415 & 3.3960 & 3.395995 \\
\cline { 2 - 7 } & 20.769 & 20.769043 & 21.396 & 21.396124 & 23.553 & 23.552729 \\
\cline { 2 - 7 } & 60.415 & 60.415453 & 61.042 & 61.042332 & 63.397 & 63.397027 \\
\hline
\end{tabular}

Table 7. Non-dimensional frequencies for $m=4, n=2$

\begin{tabular}{|c|c|c|c|c|c|c|}
\hline \multirow{3}{*}{$\beta$} & \multicolumn{6}{|c|}{$c$} \\
\hline & \multicolumn{2}{|c|}{0.1} & \multicolumn{2}{|c|}{0.3} & \multicolumn{2}{|c|}{0.7} \\
\hline & Wang [13] & PSM & Wang [13] & PSM & Wang [13] & PSM \\
\hline \multirow{3}{*}{0} & 3.6737 & 3.673701 & 4.0669 & 4.066932 & 5.5093 & 5.509268 \\
\hline & 21.550 & 21.550253 & 20.556 & 20.555506 & 18.641 & 18.641218 \\
\hline & 58.189 & 59.188637 & 54.015 & 54.015186 & 42.810 & 42.810666 \\
\hline \multirow{3}{*}{2.5} & 3.0821 & 3.082123 & 3.5181 & 3.518063 & 5.0536 & 5.053637 \\
\hline & 21.062 & 21.062033 & 20.085 & 20.084902 & 18.212 & 18.212036 \\
\hline & 58.693 & 58.692572 & 53.543 & 53.543037 & 42.385 & 42.385379 \\
\hline \multirow{3}{*}{7.5} & 1.2137 & 1.213739 & 2.0036 & 2.003562 & 3.9836 & 3.983635 \\
\hline & 20.048 & 20.048376 & 19.108 & 19.107981 & 17.322 & 17.321630 \\
\hline & 57.686 & 57.685602 & 57.584 & 57.584453 & 41.521 & 41.521269 \\
\hline
\end{tabular}

\section{Conclusions}

Typically, the free vibration frequencies of a non-uniform gravity loaded Euler-Bernoulli column/beam is obtained numerically as the governing fourth order differential equation with variable coefficients does not yield any closed form solutions. The numerical technique-Chebyshev Pseudospectral method explored in this paper introduces a novel formulation of the method in which the basis functions and their derivatives are computed using 
trigonometric functions. The stability of the method is first studied by obtaining the vibration frequencies of a linearly tapered beam in the absence of gravity. The accuracy of the method is further tested against the exact solutions of a class of non-uniform beams in the absence of gravity, solutions obtained using two variants of DQM for a non-uniform column under different end conditions and also against the solutions obtained using an initial value method in the case of a uniform column under self-weight. Finally, the proposed method is used to find the vibration frequencies of a standing linearly tapered heavy column. A comparison of the results obtained with those of the numerical initial value method shows that the proposed technique is an efficient and reliable method in handling vibration columns of elastic columns/beams. It is also possible to extend the technique to other tapers and consider inclusion of shear effects as in a Timoshenko column.

\section{References}

[1] Wei D. J., Yan S. X., Zhang Z. P., Li X.-F. Critical load for bukling of non-prismatic columns under self-weight and tip force. Mechanical Research and Communications, Vol. 37, 2010, p. 554-558.

[2] Cifuentes J., Kapania R. K. A comparison of FEM and semi-analytical method in the buckling and vibration of non-prismatic columns under tip force and self-weight. 56th AIAA/ASCE/AHC/ASC Structures, Structural Dynamics and Materials Conference, Florida, 2015.

[3] Duan W. H., Wang C. M. Exact Solution of buckling of columns including self-weight. ASCE Journal of Engineering Mechanics, Vol. 134, 2008, p. 116-119.

[4] Wang C. Y. Vibration of a segmented heavy column. Journal of Vibration and Acoustics, Vol. 132, 2010, p. 1-4.

[5] Greenhill A. G. Determination of the greatest height consistent with stability that a vertical pole or mast must be made, and of the greatest height to which a free of given population can grow. Proceedings of Combridge Philsophycal Society, Vol. 4, Issue 2, 1881, p. 65-73.

[6] Schafer B. Free vibration of a gravity loaded calmped-free beam. Ingenieur-Archiv, Vol. 55, 1985, p. 66-80.

[7] Yokoyama T. Vibrations of a hanging Timoshenko beam under gravity. Journal of Sound and Vibration, Vol. 141, Issue 2, 1990, p. 245-258.

[8] Virgin L. N., Santillan S. T., Holland D. B. Effect of gravity on the vibration of vertical Cantilevers. Mechanical Research and Communications, Vol. 34, 2007, p. 312-317.

[9] Okay F., Atay M. T., Coskun S. B. Determination of buckling loads and mode shapes of a heavy vertical column under its own weight using the variational iteration method. International Journal of Nonlinear Sciences and Numerical Simulation, Vol. 11, Issue 10, 2010, p. 851-857.

[10] Huang Y., Li X.-F. Buckling analysis of non-uniform and axially graded columns with varying flexural rigidity. ASCE Journal of Engineering Mechanics, Vol. 137, 2011, p. 73-81.

[11] Mahmoud A. A., Awadalla R., Nassar M. M. Free vibration of non-uniform column using DQM. Mechanical Research and Communications, Vol. 38, 2011, p. 443-448.

[12] Taha M., Essam M. Stability behavior and free vibration of tapered columns with elastic end restraints using the DQM method. Ain Shams Engineering Journal, Vol. 4, 2013, p. 515-521.

[13] Wang C. Y. Influence of gravity and taper on the vibration of a standing column. Advances in Applied Mathematics and Mechanics, Vol. 4, Issue 4, 2012, p. 483-495.

[14] Lee J., Schultz W. W. Eigenvalue analysis of Timoshenko beams and axisymmetric midlin plates by the pseudospectral method. Journal of Sound and Vibration, Vol. 269, 2004, p. 609-621.

[15] Yagci B., Filiz S., Romero L. L., Ozdoganlar O. B. A spectral Tchebyshev technique for solving linear and nonlinear beam equations. Journal of Sound and Vibration, Vol. 321, 2004, p. 375-404.

[16] Sari M. S., Butcher E. A. Free vibration of non-rotating and rotating Timoshenko beams with damaged boundaries using the Chebyshev collocation method. International Journal of Mechanical Sciences, Vol. 60, 2012, p. 1-11.

[17] Wattanasakulpong N., Mao Q. Dynamic response of Timoshenko functionally graded beams with classical and non-classical boundary conditions using Chebyshev collocation method. Composite Structures, Vol. 119, 2015, p. 346-354.

[18] Masjedi P. K., Ovesy H. R. Chebyshev collocation method for static intrinsic equations of geometrically exact beams. International Journal of Solids and Structures, Vol. 54, 2015, p. 183-191. 
[19] Barasch S., Chen Y. On the vibration of a rotating disk. Journal of Applied Mechanics, Vol. 39, 1972, p. 1143-1144.

[20] Wang C. Y., Wang C. M. Exact vibration solutions for a class of nonuniform beams. Journal of Engineering Mechanics, Vol. 139, 2013, p. 928-931.

[21] Sarkar K., Ganguli R., Ghosh D., Elisahakoff I. Closed form solutions and uncertainty quantification for gravity-loaded beams. Meccanica, Vol. 51, 2016, p. 1465-1479.

[22] Boyd J. P. Chebyshev and Fourier Spectral Methods. Dover, New York, USA, 2001.

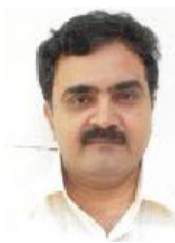

Gopinathan Sudheer received his Ph.D. degree in applied mathematics from Andhra University, Visakhapatnam, India in 2006. He is working as Professor in Department of Mathematics, GVP College of Engineering for Women, Visakhapatnam, India. His current research interests include vibration analysis, ultrasonic NDT, pseudospectral methods and forecasting

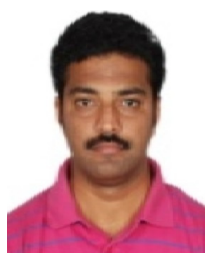

P. Sri Harikrishna received his M.Phil. degree in applied mathematics from Andhra University, India in 2005. He is working as Assistant Professor in Department of Applied Mathematics, GITAM Institute of Science, GITAM University, Visakhapatnam, India. His current research interests include pseudospectral methods.

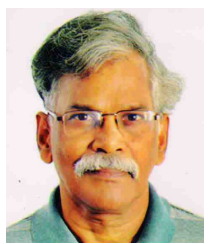

Y. Vasudeva Rao received Ph.D. degree in applied mathematics from Andhra University, Visakhapatnam, India in 1980. He was with Faculty of Applied Mathematics, Andhra University from 1972-2009. From 2010 he is visiting faculty, School of Basic Sciences, IIT Bhubaneswar. His current research interests include techniques of applied mathematics-analytical and numerical and applied continuum mechanics 